\title{
Para-Grassmannian Coherent and Squeezed States for Pseudo-Hermitian $q$-Oscillator and their Entanglement
}

\author{
Yusef MALEKI \\ Department of Physics, University of Mohaghegh Ardabili, Ardabil, 179, Iran \\ E-mail: maleki.thph1365@yahoo.com
}

Received May 27, 2011, in final form August 19, 2011; Published online August 25, 2011

http://dx.doi.org/10.3842/SIGMA.2011.084

\begin{abstract}
In this paper, $q$-deformed oscillator for pseudo-Hermitian systems is investigated and pseudo-Hermitian appropriate coherent and squeezed states are studied. Also, some basic properties of these states is surveyed. The over-completeness property of the paraGrassmannian pseudo-Hermitian coherent states (PGPHCSs) examined, and also the stability of coherent and squeezed states discussed. The pseudo-Hermitian supercoherent states as the product of a pseudo-Hermitian bosonic coherent state and a para-Grassmannian pseudoHermitian coherent state introduced, and the method also developed to define pseudoHermitian supersqueezed states. It is also argued that, for $q$-oscillator algebra of $k+1$ degree of nilpotency based on the changed ladder operators, defined in here, we can obtain deformed $S U_{q^{2}}(2)$ and $S U_{q^{2 k}}(2)$ and also $S U_{q^{2 k}}(1,1)$. Moreover, the entanglement of multilevel para-Grassmannian pseudo-Hermitian coherent state will be considered. This is done by choosing an appropriate weight function, and integrating over tensor product of PGPHCSs.
\end{abstract}

Key words: para-Grassmann variables; coherent state; squeezed state; pseudo-Hermiticity; entanglement

2010 Mathematics Subject Classification: 81R30; 15A75; 81P40

\section{Introduction}

The study of non-Hermitian Hamiltonians with real spectra have attracted a great deal of attention in the last decade $[1,2,3,4,5,6]$. Bender and his coauthors $[2,3]$ found examples of nonHermitian Hamiltonians with real spectra. Since these Hamiltonians were invariant under $\mathcal{P} T$ transformations, their spectral properties were considered to be related to their $\mathcal{P} T$-symmetry. Later, the basic structure responsible for the reality of the spectrum of a non-Hermitian Hamiltonian was explored by Mostafazadeh, and he mentioned that pseudo-Hermiticity is the reason for the particular spectral properties of these Hamiltonians, he also investigated its various consequences and its connection with $\mathcal{P} T$-symmetric quantum systems and pointed out that all the $\mathcal{P} T$-symmetric non-Hermitian Hamiltonians studied in the literature belong to the class of pseudo-Hermitian Hamiltonians. Consequently, in the framework of non-Hermitian systems, the notion of pseudo-Hermitian operator as a non-Hermitian operator with real spectrum has been found to have a considerable importance $[5,6]$.

In the same direction, the big number of works on coherent states and their various applications, indicates an excited interest of today's researchers in this field $[7,8,9,10,11,12$, 13, 14, 15, 16, 17, 18, 19]. Among all, Grassmannian coherent states and their generalizations have been widely investigated in recent years, and various applications of Grassmann variables have been studied in theoretical physics and quantum optics [16, 17, 19, 20, 21]. In [22], the authors focused on the $q$-oscillator algebra and introduced appropriate coherent states based on para-Grassmann variables. Recently, some attempts have been made to develop the study 
of coherent states to pseudo-Hermitian quantum systems, pseudo-Hermitian coherent state for bosonic systems, and pseudo-Hermitian coherent state for two-level and $n$-level systems based on Grassmann numbers have been studied [23, 24, 25]. In the present paper, $q$-deformed oscillator for pseudo-Hermitian systems will be investigated and pseudo-Hermitian appropriate coherent states will be considered. The pursued method in defining coherent states here is inspired by the definition in [22]. The over-completeness property of these pseudo-Hermitian para-Grassmannian pseudo-Hermitian coherent states (PGPHCSs) will be examined, and using the definition of the coherent states for pseudo-Hermitian bosonic systems, pseudo-Hermitian supercoherent states as the product of a pseudo-Hermitian bosonic coherent state by a pseudoHermitian para-Grassmannian coherent state will be introduced [26, 27, 28, 18]. The method also will be developed to pseudo-Hermitian squeezed states and pseudo-Hermitian supersqueezed states. It will also be argued that for $q$-oscillator algebra of $k+1$ degree of nilpotency based on the changed ladder operators, defined in here we can develop deformed $S U_{q^{2}}(2)$ and $S U_{q^{2 k}}(2)$ and also $S U_{q^{2 k}}(1,1)$. One of the aims of the present work is to consider entanglement of multilevel PGPHCSs [29, 30]. This is the generalization of the work done in [10, 11] to the PGPHCSs. Similar to what we have done in those papers, choosing some appropriate weight functions, we conclude that it is possible to construct some entangled pure states for pseudo-Hermitian systems [10, 11]. The pseudo-Hermitian version of the Bell, W and GHZ will be constructed. Therefore, the PGPHCSs can be applied in quantum information and quantum computation.

This paper is organized as follows. In Section 2, the concept of a pseudo-Hermitian operator is introduced and the basic spectral properties of pseudo-Hermitian Hamiltonians is given. In Section 3, the definition and basic properties of the para-Grassmann variables and $q$-oscillator algebra is considered. Then the notion of pseudo-Hermitian $q$-oscillator algebra and their properties is given and also, the appropriate PGPHCSs are introduced. It is shown that unlike the canonical Hermitian coherent states, PGPHCSs satisfy bi-over-completeness condition instead of over-completeness one. The time evolution and stability of the PGPHCSs are investigated. Also the construction of pseudo-Hermitian supercoherent states is studied. Finally modified $S U_{q^{2}}(2)$ and $S U_{q^{2 k}}(2)$ and also $S U_{q^{2 k}}(1,1)$ are introduced. In Section 4, the definition of squeezed states and their stability and supersqueezed states are argued. Section 5 is devoted to the entanglement of multi-level PGPHCSs. Choosing appropriate weight functions, we construct some entangled pure states for pseudo-Hermitian systems. The pseudo-Hermitian version of the Bell, $\mathbf{W}$ and GHZ using two level PGPHCSs, is investigated. These states degenerate to the well-known Bell, W and GHZ states, when PGPHCSs reduce to the usual Hermitian coherent staes. In addition, some examples of entangled qutrit states are given. Finally, the method is developed for qudit case. A brief conclusion is given in section 6 .

\section{Pseudo-Hermitian operators}

In this section, the notion of the pseudo-Hermiticity of operators is considered. In fact the necessary and sufficient condition for the reality of the spectrum of a non-Hermitian Hamiltonian admitting a complete set of biorthonormal eigenvectors is related to their pseudo-Hermiticity. In order to define the pseudo-Hermitian operator, consider $H: \mathcal{H} \rightarrow \mathcal{H}$, to be a linear operator acting in a Hilbert space $\mathcal{H}$ and $\eta: \mathcal{H} \rightarrow \mathcal{H}$, as a linear Hermitian automorphism. Then the operator

$$
H^{\sharp}=\eta^{-1} H^{\dagger} \eta,
$$

is defined as the $\eta$-pseudo-Hermitian adjoint of $H$. The operator $\mathrm{H}$ is said to be $\eta$-pseudoHermitian (with respect to $\eta$ ) if $H^{\sharp}=H$. If $\mathrm{H}$ is taken to be a pseudo-Hermitian diagonalizable linear operator then, the eigenvalues are real or come in complex conjugate pairs and the 
multiplicity of complex conjugate eigenvalues are the same (to review the basic properties of pseudo-Hermitian operators see $[4,5,6])$. According to [5] considering diagonalizable operators $H$ with discrete spectrum, a complete set of biorthonormal eigenvectors $\left\{\left|\psi_{i}\right\rangle,\left|\varphi_{i}\right\rangle\right\}$ exists such that

$$
\begin{aligned}
& H\left|\psi_{i}\right\rangle=E_{i}\left|\psi_{i}\right\rangle, \quad H^{\dagger}\left|\varphi_{i}\right\rangle=\bar{E}_{i}\left|\varphi_{i}\right\rangle, \\
& \left\langle\varphi_{i} \mid \psi_{i}\right\rangle=\delta_{i j}, \quad \sum_{i}\left|\psi_{i}\right\rangle\left\langle\varphi_{i}\left|=\sum_{i}\right| \varphi_{i}\right\rangle\left\langle\psi_{i}\right|=I .
\end{aligned}
$$

In fact, any non-Hermitian Hamiltonian with a discrete real spectrum and a complete biorthonormal system of eigenbasis vectors is pseudo-Hermitian. Considering the above discussion, for a nondegenerate case, the operator $\eta$ and it's inverse satisfy the (2.1) by taking

$$
\eta=\sum_{i}\left|\varphi_{i}\right\rangle\left\langle\varphi_{i}\left|, \quad \eta^{-1}=\sum_{i}\right| \psi_{i}\right\rangle\left\langle\psi_{i}\right|,
$$

thus one has

$$
\left|\varphi_{i}\right\rangle=\eta\left|\psi_{i}\right\rangle, \quad\left|\psi_{i}\right\rangle=\eta^{-1}\left|\varphi_{i}\right\rangle
$$

It is notable that $\eta$ is not unique, however it can be expressed as above.

\section{$3 \quad$ Pseudo-Hermitian $q$-oscillator and coherent states}

\subsection{Para-Grassmann variables and $q$-oscillator algebra}

Grassmann variables and their different generalizations along with their applications have been widely surveyed and the mathematical structure of these non-commuting mathematical objects were studied in resent years $[18,19,22,31,32,33,34,35,36]$. To define para-Grassmann variables, consider the non-commutative variables $\theta$ and $\bar{\theta}$, which satisfy the following relations [22]

$$
\theta^{p+1}=0, \quad \bar{\theta}^{p+1}=0, \quad \theta \bar{\theta}=q^{2} \bar{\theta} \theta, \quad \text { where } \quad q^{2}=e^{\frac{2 \pi i}{p+1}},
$$

in the above relations, $p$ is a non-zero integer number. The integral calculus appropriate for these variables is

$$
\int d \theta \theta^{n}=\delta_{n, p} \sqrt{[p] !}, \quad \text { where } \quad[X]=\frac{q^{2 X}-1}{q^{2}-1}, \quad[n] !=[n] \cdots[1] .
$$

The $q$-deformed oscillator satisfies the following relations [18, 37, 38]

$$
a a^{\dagger}-q a^{\dagger} a=q^{-N}, \quad a a^{\dagger}-q^{-1} a^{\dagger} a=q^{N} .
$$

We note that annihilation and creation operators have the nilpotency degree of order $p+1$.

\subsection{Pseudo-Hermitian $q$-oscillator algebra}

In analogy with the above mentioned $q$-oscillator algebra for Hermitian systems, let us define pseudo-Hermitian $q$-oscillator algebra as follows

$$
a a^{\sharp}-q a^{\sharp} a=q^{-N}, \quad a a^{\sharp}-q^{-1} a^{\sharp} a=q^{N},
$$

and also, considering the dual space we have

$$
\tilde{a} a^{\dagger}-q a^{\dagger} \tilde{a}=q^{-N}, \quad \tilde{a} a^{\dagger}-q^{-1} a^{\dagger} \tilde{a}=q^{N},
$$


where $a$ is creation operator for pseudo-Hermitian $q$-deformed oscillator and can be expressed as follows

$$
a:=\sum_{n=0}^{p} \sqrt{[[n+1]]}\left|\psi_{n}\right\rangle\left\langle\phi_{n+1}|\quad \Longrightarrow \quad a| \psi_{n}\right\rangle=\sqrt{[[n]]}\left|\psi_{n-1}\right\rangle,
$$

and let us define the creation operator for the pseudo-Hermitian $q$-deformed oscillator in the Fock space spanned by the basis $\left\{\left|\psi_{0}\right\rangle \cdots\left|\psi_{n}\right\rangle\right\}$ as

$$
a^{\sharp}:=\eta^{-1} a^{\dagger} \eta:=\sum_{n=0}^{p} \sqrt{[[n+1]]}\left|\psi_{n+1}\right\rangle\left\langle\phi_{n}\right|,
$$

where, we have

$$
[[X]]=\frac{q^{X}-q^{-X}}{q-q^{-1}} .
$$

Considering the dual space, one can define the creation and annihilation operators which act on dual states. In analogy with annihilation operator $a$, let define

$$
\tilde{a}:=\eta a \eta^{-1}:=\sum_{n=0}^{p} \sqrt{[[n+1]]}\left|\phi_{n}\right\rangle\left\langle\psi_{n+1}\right| .
$$

Hence, $a^{\sharp}$ is $\eta$-pseudo-adjoint to $a$ and $\tilde{a}$ is $\eta^{-1}$-pseudo-adjoint to $a^{\dagger}$. So, the operator $a^{\dagger}$ for pseudo-Hermitian $q$-deformed oscillator can be written as

$$
a^{\dagger}:=\sum_{n=0}^{p} \sqrt{[[n+1]]}\left|\phi_{n+1}\right\rangle\left\langle\psi_{n}\right|
$$

Thus one has

$$
a^{\sharp} a=[[N]] \quad \Longrightarrow \quad[[N]]^{\sharp}=\eta^{-1}[[N]]^{\dagger} \eta=[[N]],
$$

and by choosing $q^{2}=e^{\frac{2 \pi i}{p+1}}$ we obtain

$$
N=\frac{p+1}{\pi} \arcsin \left(a^{\sharp} a \sin \frac{\pi}{p+1}\right) .
$$

Hence, the action of the operator $N$ on $\left|\psi_{n}\right\rangle$ reads

$$
N\left|\psi_{n}\right\rangle=n\left|\psi_{n}\right\rangle .
$$

Furthermore, we get the commutation relations between the annihilation and creation operators and the operator $N$ as

$$
[N, a]=-a, \quad\left[N, a^{\sharp}\right]=-a^{\sharp} .
$$

For the dual space operators we can write

$$
a^{\dagger} \tilde{a}=\left[\left[N^{\prime}\right]\right] \quad \Longrightarrow \quad\left[\left[N^{\prime}\right]\right]^{\sharp}=\eta\left[\left[N^{\prime}\right]\right]^{\dagger} \eta^{-1}=\left[\left[N^{\prime}\right]\right] .
$$

Again, by choosing $q^{2}=e^{\frac{2 \pi i}{p+1}}$ we obtain

$$
N^{\prime}=\frac{p+1}{\pi} \arcsin \left(a^{\dagger} \tilde{a} \sin \frac{\pi}{p+1}\right) .
$$




\subsection{Change of variables and coherent states}

Now consider the following change of variables

$$
b=q^{\frac{N}{2}} a, \quad \bar{b}=a^{\sharp} q^{\frac{N}{2}} \quad \text { and } \quad c=q^{\frac{N^{\prime}}{2}} \tilde{a}, \quad \bar{c}=a^{\dagger} q^{\frac{N^{\prime}}{2}} .
$$

These new operators are necessary for the forthcoming purposes throughout the paper, also the coherent and squeezed states will be defined based on these operators not $a$ and $a^{\sharp}$ (also $\tilde{a}$ and $a^{\dagger}$ ). Taking into account these variables we see that

$$
b \bar{b}-q^{2} \bar{b} b=1, \quad \bar{b} b-b \bar{b}=q^{2 N}, \quad \bar{b} b=[N],
$$

and for the dual operators we get

$$
c \bar{c}-q^{2} \bar{c} c=1, \quad \bar{c} c-c \bar{c}=q^{2 N}, \quad \bar{c} c=[N],
$$

We not that in [25] the creation operator $b^{\sharp}$ is $\eta$-pseudo-Hermitian adjoint of the annihilation operator $b$, but our changed ladder operator $\bar{b}$ is not $\eta$-pseudo-Hermitian adjoint of $b$, and we have $b^{\sharp}=\bar{b} q^{-N}$. One could easily check that the pseudo-Hermitian number states can be expressed in terms of $b$ and $\bar{b}$ (also $c$ and $\bar{c}$ for the dual space), and these operators annihilate and create the number states as follows

$$
\left|\psi_{n}\right\rangle=\frac{(\bar{b})^{n}}{\sqrt{[n] !}}\left|\psi_{0}\right\rangle, \quad b\left|\psi_{n}\right\rangle=\sqrt{[n]}\left|\psi_{n-1}\right\rangle, \quad \bar{b}\left|\psi_{n}\right\rangle=\sqrt{[n+1]}\left|\psi_{n}\right\rangle .
$$

Thus

$$
[N, b]=-b, \quad[N, \bar{b}]=\bar{b}, \quad[N, c]=-c, \quad[N, \bar{c}]=\bar{c}
$$

For the future uses, we need to consider the commutation relation between these operators and para-Grassmannian variables

$$
[\theta, b]_{q^{2}}=0, \quad[\bar{b}, \theta]_{q^{2}}=0, \quad[\bar{\theta}, \bar{b}]_{q^{2}}=0, \quad[b, \bar{\theta}]_{q^{2}}=0
$$

where the $p$-commutator is defined as

$$
[A, B]_{p}=A B-p B A \text {. }
$$

Let us have the following quantization relations between the biorthonormal pseudo-Hermitian eigenstates $\left\{\left|\psi_{n}\right\rangle,\left|\phi_{n}\right\rangle\right\}$ and para-Grassmannian variables

$$
\begin{array}{llrl}
\theta\left|\psi_{n}\right\rangle & =q^{-2 n}\left|\psi_{n}\right\rangle \theta, & & \bar{\theta}\left\langle\psi_{n}\right|=q^{-2 n}\left\langle\psi_{n}\right| \bar{\theta}, \\
\theta\left\langle\psi_{n}\right|=q^{2 n}\left\langle\psi_{n}\right| \theta, & & \bar{\theta}\left|\psi_{n}\right\rangle=q^{2 n}\left|\psi_{n}\right\rangle \bar{\theta}, \\
\theta\left|\phi_{n}\right\rangle=q^{-2 n}\left|\phi_{n}\right\rangle \theta, & & \bar{\theta}\left\langle\phi_{n}\right|=q^{-2 n}\left\langle\phi_{n}\right| \bar{\theta}, \\
\theta\left\langle\phi_{n}\right|=q^{2 n}\left\langle\phi_{n}\right| \theta, & & \bar{\theta}\left|\phi_{n}\right\rangle=q^{2 n}\left|\phi_{n}\right\rangle \bar{\theta} .
\end{array}
$$

Now let $|\theta\rangle$ be a PGPHCS, then following the definition of the canonical Hermitian coherent state, the PGPHCS $|\theta\rangle$ can be defined as the eigenstates of annihilation operator $b$ for the space spanned by the basis $\left\{\left|\psi_{n}\right\rangle\right\}$. Therefore, PGPHCS $|\theta\rangle$ reads

$$
b|\theta\rangle=\theta|\theta\rangle .
$$


In order to find the explicit form of the PGPHCS $|\theta\rangle$, we can generically write

$$
|\theta\rangle=\sum_{n=0}^{p} c_{n} \theta^{n}\left|\psi_{n}\right\rangle
$$

The two equations (3.3) and (3.4) give

$$
|\theta\rangle=\sum_{n=0}^{p} \frac{q^{n(n+1)}}{\sqrt{[n] !}} \theta^{n}\left|\psi_{n}\right\rangle=e_{q}^{\bar{b} \theta}\left|\psi_{0}\right\rangle,
$$

we can interpret $D(\theta)=e_{q}^{\bar{b} \theta}$ as the displacement operator for the PGPHCS. We note that $q$-deformed exponential is

$$
e_{q}^{x}=\sum_{n=0}^{p} \frac{x^{n}}{[n] !}
$$

We can easily check that the action of the creation operator $\bar{b}$ on the coherent state gives

$$
\bar{b}|\theta\rangle=q^{-2} \sum_{n=1}^{p} q^{n(n+1)} \frac{[n]}{\sqrt{[n] !}} \theta^{n-1}\left|\psi_{n}\right\rangle .
$$

The overlap between the state $\left|\phi_{n}\right\rangle$ and the coherent state is given as

$$
\left\langle\phi_{n} \mid \theta\right\rangle=\frac{q^{-n(n-1)}}{\sqrt{[n] !}} \theta^{n} .
$$

We can also define the other set of coherent states based on the creation operator $\bar{b}$ with paraGrassmanian eigenvalue as follows

$$
\langle\bar{\theta}| \bar{b}=\langle\bar{\theta}| \bar{\theta} .
$$

Thus, in the terms of number state basis the coherent states of the creation operator $\bar{b}$ can be expanded as

$$
\langle\bar{\theta}|=\sum_{n=0}^{p} \frac{q^{n(n-1)}}{\sqrt{[n] !}} \bar{\theta}^{n}\left\langle\psi_{n}\right| .
$$

Similar to (3.6), the overlap between the state $\left|\phi_{n}\right\rangle$ and this coherent state depends on $n$ and takes the following form

$$
\left\langle\bar{\theta} \mid \phi_{n}\right\rangle=\frac{q^{n(n-1)}}{\sqrt{[n] !}} \bar{\theta}^{n} .
$$

As was seen, for the pseudo-Hermitian systems, there are two sets of annihilation and creation operators, with respect to the basis $\left\{\left|\psi_{n}\right\rangle\right\}$ and $\left\{\left|\phi_{n}\right\rangle\right\}$ respectively. Therefore, a coherent state can be defined in the space spanned by the basis $\left\{\left|\phi_{n}\right\rangle\right\}$, which is the eigenstates of annihilation operator $c$ with para-Grassmanian eigenvalue $\theta$ similar to what we had for the operator $b$, as follows

$$
c \widetilde{\theta\rangle}=\theta \widetilde{|\theta\rangle} .
$$


Again taking the general form of the coherent state in (3.4) and using the above relation for the coherent states, it is straightforward to see that the coherent state is

$$
\widetilde{|\theta\rangle}=\sum_{n=0}^{p} \frac{q^{n(n+1)}}{\sqrt{[n] !}} \theta^{n}\left|\phi_{n}\right\rangle=e_{q}^{\bar{c} \theta}\left|\phi_{0}\right\rangle .
$$

Moreover, by acting the creation operator $\bar{c}$ on coherent state we get

$$
\bar{c}|\theta\rangle=q^{-2} \sum_{n=1}^{p} q^{n(n+1)} \frac{[n]}{\sqrt{[n] !}} \theta^{n-1}\left|\phi_{n}\right\rangle .
$$

The overlap between the state $\left|\psi_{n}\right\rangle$ and the coherent state of the annihilation operator $c$ is proportional to $\theta^{n}$ as

$$
\left\langle\psi_{n} \widetilde{|\theta\rangle}=\frac{q^{-n(n-1)}}{\sqrt{[n] !}} \theta^{n} .\right.
$$

Hence, the overlap between the state $\left|\phi_{n}\right\rangle$ and (3.5) is equal to the overlap between the state $\left|\psi_{n}\right\rangle$ and the coherent state of the annihilation operator $c$ defined above, i.e., $\left\langle\bar{\theta} \mid \phi_{n}\right\rangle=\left\langle\psi_{n} \mid \widetilde{\theta}\right\rangle$. Furthermore, one may define the coherent state of the creation operator $\bar{c}$, as the superposition of the number states of the dual space with para-Grassmanian eigenvalue $\bar{\theta}$ as

$$
\widetilde{\langle\bar{\theta}| \bar{c}}=\widetilde{\langle\bar{\theta}|} \bar{\theta}
$$

Again we can expand the coherent state based on the number states of the related space and powers of the para-Grassmanian variable $\bar{\theta}$ as

$$
\widetilde{\langle\bar{\theta}|}=\sum_{n=0}^{p} \frac{q^{n(n-1)}}{\sqrt{[n] !}} \bar{\theta}^{n}\left\langle\phi_{n}\right| .
$$

Hence, we have

$$
\widetilde{\langle\bar{\theta}}\left|\psi_{n}\right\rangle=\frac{q^{n(n-1)}}{\sqrt{[n] !}} \bar{\theta}^{n} .
$$

Consequently, there is a relation between the overlaps of coherent states with number states as $\left\langle\bar{\theta} \mid \phi_{n}\right\rangle=\widetilde{\bar{\theta}}\left|\psi_{n}\right\rangle$. So, there is a relation between the overlaps of the coherent states of the annihilation operators with number states of their dual states and also between the overlaps of the coherent states of the creation operators with number states of their dual states as well.

\subsection{Resolution of identity}

Here, the over-completeness property of the states $|\theta\rangle$ and $\widetilde{|\theta\rangle}$ is examined. By introducing the generic form of the weight function as

$$
w(\theta, \bar{\theta})=\sum_{k, l=0}^{n-1} c_{k l} \theta^{k} \bar{\theta}^{l} .
$$

We see that the integrals of the terms $|\theta\rangle\langle\theta|$ and $\widetilde{|\theta\rangle} \widetilde{\langle\theta|}$ do not satisfy over-completeness relation. Hence, in order to realize the resolution of identity, it is necessary to consider the biorthonormal nature of the pseudo-Hermitian systems. So, as done in [24, 25], it is reasonable to check the 
integrals $|\theta\rangle \widetilde{\langle\theta|}$ and $\widetilde{|\theta\rangle}\langle\theta|$ with the measure $d \bar{\theta} d \theta w(\theta, \bar{\theta})$. Choosing the proper weight function, we can write the resolution of identity as

$$
\int d \bar{\theta} d \theta w(\theta, \bar{\theta})|\theta\rangle \widetilde{\langle\theta|}=\int d \bar{\theta} d \theta w(\theta, \bar{\theta}) \widetilde{|\theta\rangle}\langle\theta|=I .
$$

To obtain the explicit form of the weight function we put $|\theta\rangle$ and $\widetilde{\langle\theta|}$ into (3.7) and get

$$
\int d \bar{\theta} d \theta w(\theta, \bar{\theta})|\theta\rangle \widetilde{\langle\theta|}=\int d \bar{\theta} d \theta \sum_{k, l=0}^{p} c_{k, l} \theta^{k} \bar{\theta}^{l} \sum_{n, m=0}^{p} \frac{q^{n(n+1)}}{\sqrt{[n] !}} \theta^{n}\left|\psi_{n}\right\rangle\left\langle\phi_{m}\right| \bar{\theta}^{m} \frac{\bar{q}^{m(m+1)}}{[m] !} .
$$

Accounting for the quantization and the integration rules of para-Grassmann variables, and utilizing the completeness of the biorthonormal basis of the pseudo-Hermitian system, i.e., $\sum_{n=0}^{p}\left|\psi_{n}\right\rangle\left\langle\phi_{n}\right|=I$, we can determine the coefficients $c_{k l}$ as

$$
c_{k, l}=\frac{[p-l] !}{[p] !} q^{-2 l(l+1)} \delta_{k, l}
$$

Thus the weight function reads

$$
w(\theta, \bar{\theta})=\sum_{l=0}^{p} \frac{[p-l] !}{[p] !} q^{-2 l(l+1)} \theta^{l} \bar{\theta}^{l} .
$$

Consequently the coherent states $|\theta\rangle$ and $\widetilde{|\theta\rangle}$ provide the bi-over-completeness relation for pseudoHermitian systems of biorthnormal coherent states. Note that when the pseudo-Hermitian system reduces to the Hermitian one, the coherent state $\widetilde{|\theta\rangle}$ degenerates to $|\theta\rangle$. It is in accordance with the over-completeness property of the Hermitian systems.

\subsection{Time evolution of PGPHCS}

In this subsection, the condition for the stability of the PGPHCS will be investigated. It will be shown that the coherent states remain eigenstate of the annihilation operator $b$, for any time evolution of the initial coherent state $|\theta, 0\rangle \equiv|\theta\rangle$, i.e., $|\theta, t\rangle$, taking a proper condition. This means that

$$
b|\theta, t\rangle=\theta(t)|\theta, t\rangle, \quad \text { where } \quad|\theta, t\rangle=e^{-i H t}|\theta\rangle .
$$

Taking the explicit form of the coherent state $|\theta\rangle$, one can write

$$
|\theta, t\rangle=\sum_{k=0}^{p} \frac{q^{k(k+1)}}{\sqrt{[k] !}} \theta^{k} e^{-i E_{k} t}\left|\psi_{k}\right\rangle
$$

Now we note that, since $k$ changes, all the energies related to the various $k$ s form the set $\left\{E_{0}, E_{1}, \ldots, E_{p}\right\}$, which are real parameters. Thus, in general we can write

$$
E_{k}=\left(k c_{k}+1\right) E_{0} .
$$

Now, if $c_{k}$ is taken to be constant $\left(c_{i}=c_{j}=c\right)$, the evolved coherent state reads

$$
|\theta, t\rangle=e^{-i E_{0} t}|\theta(t)\rangle, \quad \text { where } \quad \theta(t)=e^{-i c E_{0} t} \theta .
$$

So, we obtain the condition that the evolved coherent state remain eigenstate of the annihilation operator $b$. This result shows that the time evolved pseudo-Hermitian $q$-deformed oscillator coherent state $|\theta\rangle$ is stable. The similar discussion holds for $|\widetilde{\theta, t}\rangle$ 's, and we can also see that $|\theta, t\rangle$ and $|\widetilde{\theta, t}\rangle$ satisfy the resolution of identity. 


\subsection{Supercoherent states}

Supersymmetric quantum mechanics needs two degrees of freedom: one described by a complex variable and the other described by a Grassmann variable. Here, the notion of pseudo-Hermitian supercoherent states will be developed. In order to provide a suitable supersymmetric Hamiltonian, we can follow the results of supersymmetric Hamiltonian for Hermitian systems based on generalized Weyl-Heisenberg algebra [26, 27, 39]. In what follows, the pseudo-Hermitian supersymmetric Hamiltonian (PHSUSH) for two level systems will be established [40]. To do so, we use pseudo-boson annihilation and creation operators (see [23]). According to [23], if a and $\mathrm{a}^{\dagger}$ are taken to be usual annihilation and creation operators for a bosonic system, then the $\eta^{\prime}$-pseudo-Hermitian adjoint of a is $\mathrm{a}^{\sharp}:=\eta^{\prime-1} \mathrm{a}^{\dagger} \eta^{\prime}$, and also $\tilde{\mathrm{a}}:=\eta^{\prime} \mathrm{a} \eta^{\prime-1}$. On the other hand, for a two level system we have $b \bar{b}+\bar{b} b=1\left(b^{2}=\bar{b}^{2}=0\right.$ and $\left.q^{2}=-1\right)$. This is similar to pseudo-fermionic algebra [24]. In other words, by replacing $\bar{b}$ with $b^{\sharp}$, the pseudo-fermionic algebra can be obtained $\left(b b^{\sharp}+b^{\sharp} b=1\right)$. We note that the operators are changed, but the obtained coherent states for both systems can be the same. Therefore, based on pseudo-fermionic and pseudo-bosonic operators, one may define the PHSUSH as

$$
H_{1}=\mathrm{a}^{\sharp} \mathrm{a}+b^{\sharp} b .
$$

Regarding the fact that the bosonic operators commute with two level operators and vice versa, this Hamiltonian is $\left(\eta^{\prime} \eta\right)$-pseudo-Hermitian. Now, taking $Q=\mathrm{a}^{\sharp} b$ and $Q^{\sharp}=\mathrm{a} b^{\sharp}$, then we have

$$
H_{1}=Q Q^{\sharp}+Q^{\sharp} Q .
$$

both of the operators $Q$ and $Q^{\sharp}$ commute with Hamiltonian. To generalize the result to multilevel systems, we can replace $\bar{b}$ with $b^{\sharp}$ in general, and get the pseudo-Hermitian version of the deformed algebra $b b^{\dagger}-q^{2} b^{\dagger} b=1$ discussed in [26, 27, 39]. Again, the operators are changed, but the obtained coherent states for both systems can be the same. To see this we take $b:=$ $\sum_{n=0}^{p} \sqrt{[n+1]}\left|\psi_{n}\right\rangle\left\langle\phi_{n+1}\right|$. Therefore, taking $\bar{b} \equiv b^{\sharp}$ the supercohertent states can be developed.

The coherent states for the pseudo-Hermitian boson systems are defined as the eigenstates of the corresponding pseudo-boson annihilation operators

$$
\mathrm{a}|\alpha\rangle=\alpha|\alpha\rangle,
$$

where $\alpha$ is a complex number, and $a$ is the annihilation operator for pseudo-Hermitian bosonic harmonic oscillator. Thus, considering the definition of the coherent state in the number states space, it can be written as follows

$$
|\alpha\rangle=e^{\frac{-|\alpha|^{2}}{2}} \sum_{n=0}^{\infty} \frac{\alpha^{n}}{\sqrt{n !}}\left|\psi_{n}\right\rangle=D(\alpha)\left|\psi_{0}\right\rangle,
$$

here, $D(\alpha)$ is the displacement operator

$$
D(\alpha):=\exp \left(\alpha \mathrm{a}^{\sharp}-\alpha^{*} \mathrm{a}\right) .
$$

On the other hand for the dual space, we can define the associated pseudo-bosonic coherent state using the corresponding annihilation operator $\tilde{a}$ as

$$
\tilde{\mathrm{a}}|\alpha\rangle^{\prime}=\alpha|\alpha\rangle^{\prime} .
$$

Again, in the number states space, the coherent state can be expanded as follows

$$
|\alpha\rangle^{\prime}=e^{\frac{-|\alpha|^{2}}{2}} \sum_{n=0}^{\infty} \frac{\alpha^{n}}{\sqrt{n !}}\left|\phi_{n}\right\rangle=D^{\prime}(\alpha)\left|\phi_{0}\right\rangle,
$$


where $D^{\prime}(\alpha)$ is the displacement operator and is similar to the displacement operator defined for its dual space

$$
D^{\prime}(\alpha):=\exp \left(\alpha a^{\dagger}-\alpha^{*} \tilde{a}\right) .
$$

In what follows the supercoherent state for pseudo-Hermitian system is defined, using the above pseudo-bosonic coherent states and para-Grassmannian coherent states for the pseudo-Hermitian $q$-oscillator as follows

$$
|\alpha, \theta\rangle=|\alpha\rangle \otimes|\theta\rangle=D(\alpha, \theta)\left|\psi_{0}\right\rangle \otimes\left|\psi_{0}\right\rangle
$$

where, the displacement operator $D(\alpha, \theta)$, is the tensor product of displacement operators for bosonic and para-Grassmanian coherent states

$$
D(\alpha, \theta)=D(\alpha) \otimes D(\theta) \quad \Longrightarrow \quad D(\theta)=e_{q}^{\bar{b} \theta} .
$$

Thus we can rewrite the above super coherent state in terms of the number states as

$$
|\alpha, \theta\rangle=e^{\frac{-|\alpha|^{2}}{2}} \sum_{n=0}^{\infty} \frac{\alpha^{n}}{\sqrt{n !}}\left|\psi_{n}\right\rangle \otimes \sum_{n=0}^{p} \frac{q^{n(n+1)}}{\sqrt{[n] !}} \theta^{n}\left|\psi_{n}\right\rangle .
$$

The state $|\alpha, \theta\rangle$ is an eigenstate of the operator $a b$ with the eigenvalue $\alpha \theta$. Since for the pseudoHermitian systems we deal with two sets of basis, unlike the Hermitian coherent states, we have some other possibilities in order to define supercoherent states for these systems. For instance, we can define the following supercoherent state

$$
|\alpha, \widetilde{\theta}\rangle=|\alpha\rangle \otimes \widetilde{\theta \theta}=D(\alpha, \widetilde{\theta})\left|\psi_{0}\right\rangle \otimes\left|\phi_{0}\right\rangle,
$$

where the displacement operator $D(\alpha, \widetilde{\theta})$ reads

$$
D(\alpha, \widetilde{\theta})=D(\alpha) \otimes \widetilde{D(\theta)} \quad \Longrightarrow \quad \widetilde{D(\theta)}=e_{q}^{\bar{c} \theta} .
$$

In this case, we have used the para-Grassmanian coherent state of the dual basis, so in terms of the number states we have

$$
|\alpha, \widetilde{\theta}\rangle=e^{\frac{-|\alpha|^{2}}{2}} \sum_{n=0}^{\infty} \frac{\alpha^{n}}{\sqrt{n !}}\left|\psi_{n}\right\rangle \otimes \sum_{n=0}^{p} \frac{q^{n(n+1)}}{\sqrt{[n] !}} \theta^{n}\left|\phi_{n}\right\rangle .
$$

Likewise, one may take

$$
\left|\alpha^{\prime}, \theta\right\rangle=|\alpha\rangle^{\prime} \otimes|\theta\rangle=D\left(\alpha^{\prime}, \theta\right)\left|\phi_{0}\right\rangle \otimes\left|\psi_{0}\right\rangle,
$$

similar to the previous cases the displacement operator is

$$
D\left(\alpha^{\prime}, \theta\right)=D^{\prime}(\alpha) \otimes D(\theta),
$$

consequently, we have

$$
\left|\alpha^{\prime}, \theta\right\rangle=e^{\frac{-|\alpha|^{2}}{2}} \sum_{n=0}^{\infty} \frac{\alpha^{n}}{\sqrt{n !}}\left|\phi_{n}\right\rangle \otimes \sum_{n=0}^{p} \frac{q^{n(n+1)}}{\sqrt{[n] !}} \theta^{n}\left|\psi_{n}\right\rangle .
$$

Finally, taking the coherent states defined on the basis $\left\{\left|\phi_{n}\right\rangle\right\}$ for both bosonic and paraGrassmanian coherent state, we obtain the following supercoherent state

$$
\left|\alpha^{\prime}, \widetilde{\theta}\right\rangle=|\alpha\rangle^{\prime} \otimes \widetilde{|\theta\rangle}=D\left(\alpha^{\prime}, \widetilde{\theta}\right)\left|\phi_{0}\right\rangle \otimes\left|\phi_{0}\right\rangle
$$

similarly, by defining $D\left(\alpha^{\prime}, \widetilde{\theta}\right)=D^{\prime}(\alpha) \otimes|\widetilde{\theta}\rangle$, we can write the coherent state in terms of the number states as

$$
\left|\alpha^{\prime}, \widetilde{\theta}\right\rangle=e^{\frac{-|\alpha|^{2}}{2}} \sum_{n=0}^{\infty} \frac{\alpha^{n}}{\sqrt{n !}}\left|\phi_{n}\right\rangle \otimes \sum_{n=0}^{p} \frac{q^{n(n+1)}}{\sqrt{[n] !}} \theta^{n}\left|\phi_{n}\right\rangle .
$$




\subsection{Deformed $S U(2)_{p}$ and $S U(1,1)_{p}$ for multi-level systems}

In this subsection, using the operators $b$ and $\bar{b}$, the condition to have deformed $S U(2)$, i.e. $S U_{p}(2)$, will be considered. It will be shown that, regarding special value for $p$ which will be derived here, these operators can be generators of $s u_{p}(2)$ Lie algebra. Note that there is no condition on $p$ and it is a complex number. Now let us define

$$
b_{z}:=[b, \bar{b}]_{p}=b \bar{b}-p \bar{b} b .
$$

Considering the commutation relation between $b$ and $b_{z}$

$$
\left.\left[b_{z}, b\right]_{p}=\left[\left(1+p^{2}-p q^{2}-p\right)+\left(q^{2}+p^{2} q^{2}-\left(q^{4}+1\right) p\right) \bar{b} b\right)\right] b,
$$

to have appropriate solution, it must be proportional to the operator $b$, so we get

$$
q^{2} p^{2}-\left(q^{4}+1\right) p+q^{2}=0 \quad \Longrightarrow \quad p=q^{2} \quad \text { or } \quad p=\bar{q}^{2}
$$

Consequently one has

$$
p=q^{2} \quad \Longrightarrow \quad\left[b_{z}, b\right]_{p}=\left[b_{z}, b\right]_{q^{2}}=\left(1-q^{2}\right) b,
$$

and

$$
p=\bar{q}^{2} \quad \Longrightarrow \quad\left[b_{z}, b\right]_{p}=\left[b_{z}, b\right]_{\bar{q}^{2}}=\left(\bar{q}^{4}-\bar{q}^{2}\right) b .
$$

From the commutator of $\bar{b}$ and $b_{z}$ the same condition will be required to get proper solution, therefore we get the following $s u_{p}(2)$ deformed algebras

$$
[b, \bar{b}]_{q^{2}}=b_{z}, \quad\left[b_{z}, b\right]_{q^{2}}=\left(1-q^{2}\right) b, \quad\left[\bar{b}, b_{z}\right]_{q^{2}}=\left(1-q^{2}\right) \bar{b},
$$

and also

$$
[b, \bar{b}]_{\bar{q}^{2}}=b_{z}, \quad\left[b_{z}, b\right]_{\bar{q}^{2}}=\left(\bar{q}^{4}-\bar{q}^{2}\right) b, \quad\left[\bar{b}, b_{z}\right]_{\bar{q}^{2}}=\left(\bar{q}^{4}-\bar{q}^{2}\right) \bar{b} .
$$

Obviously, if $b^{k+1}=\bar{b}^{k+1}=0$, then $\left(q^{2}\right)^{k+1}=1$, so there are two different algebras associated with $p=q^{2}$ and $p=q^{2 k}$. Therefore, the second algebra can be written as

$$
[b, \bar{b}]_{q^{2 k}}=b_{z}, \quad\left[b_{z}, b\right]_{q^{2 k}}=\left(q^{2(k-1)}-q^{2 k}\right) b, \quad\left[\bar{b}, b_{z}\right]_{q^{2 k}}=\left(q^{2(k-1)}-q^{2 k}\right) \bar{b} .
$$

Now let define the operator $b_{z}$ in a different way as

$$
b_{z}:=[\bar{b}, b]_{p}=\bar{b} b-p b \bar{b}
$$

The commutation relation between $b$ and $b_{z}$ becomes

$$
\left[b_{z}, b\right]_{p}=\left[\left(p^{2}+q^{2} p^{2}-2 p\right)+\left(p^{2} q^{4}-2 p q^{2}+1\right) \bar{b} b\right] b,
$$

to have proper solution, the commutation relation must be independent from the operator $\bar{b} b$, therefore, the coefficient of the operator $\bar{b} b$ must vanish. this means

$$
p^{2} q^{4}-2 p q^{2}+1=0 \quad \Longrightarrow \quad\left(p q^{2}-1\right)^{2}=0 \quad \Longrightarrow \quad p=\bar{q}^{2} .
$$

Thus, in this case we have unique solution for $p$, and the above commutation relation reduces to

$$
p=\bar{q}^{2} \quad \Longrightarrow \quad\left[b_{z}, b\right]_{p}=\left[b_{z}, b\right]_{\bar{q}^{2}}=\left(\bar{q}^{4}-\bar{q}^{2}\right) b
$$


Just like the previous one, from the commutator of $\bar{b}$ and $b_{z}$ the same condition will be required in order to get proper solution. If $\left(q^{2}\right)^{k+1}=1$, then we have $p=q^{2 k}$. Consequently the algebra degenerates to

$$
[\bar{b}, b]_{q^{2 k}}=b_{z}, \quad\left[b_{z}, b\right]_{q^{2 k}}=\left(q^{2(k-1)}-q^{2 k}\right) b, \quad\left[\bar{b}, b_{z}\right]_{q^{2 k}}=\left(q^{2(k-1)}-q^{2 k}\right) \bar{b} .
$$

It must be stressed that, although this algebra is similar to (3.8), they correspond to different definitions of the operator $b_{z}$ and are not the same algebras. The difference between definition of the operator $b_{z}$ in the two algebras may recall the difference between the definition of the $s u(2)$ and $s u(1,1)$ algebras. Therefore, one may call this algebra deformed $s u(1,1)_{q^{2 k}}$ algebra. It is notable that, the above algebras were derived based on the relation between the operators $b$ and $\bar{b}$, and it would not be possible to develop similar algebras using the creation and annihilation operators $a$ and $a^{\sharp}$.

\section{Squeezed states}

By recalling the definition of the standard bosonic harmonic oscillator squeezing operator, it is worthwhile to define para-Grassmannian pseudo Hermitian squeezing operator based on the new operators for $q$-deformed oscillator as follows

$$
S(\theta)=\exp \left[\frac{1}{2}\left(\theta \bar{b}^{2}-\bar{\theta} b^{2}\right)\right] .
$$

Therefore, by applying the operator $S(\theta)$ on the vacuum state $\left|\psi_{0}\right\rangle$, the para-Grassmannian pseudo Hermitian squeezed states can be defined as

$$
|\xi\rangle=S(\theta)\left|\psi_{0}\right\rangle
$$

It is notable that the above operator is squeezing operator for any $n>2$ level systems, so for three level system (since the operators $b^{3}$ and $\bar{b}^{3}$ become zero), the squeezing operator can be expanded as

$$
S(\theta)=I+\frac{1}{2}\left(\theta \bar{b}^{2}-\bar{\theta} b^{2}\right)-\frac{\theta \bar{\theta}}{8}\left(q^{2} \bar{b}^{2} b^{2}+b^{2} \bar{b}^{2}\right) .
$$

Thus, the three level squeezed state reads

$$
|\xi\rangle=\left|\psi_{0}\right\rangle+\frac{\sqrt{[2]}}{2} \theta\left|\psi_{2}\right\rangle-\frac{[2]}{8} \theta \bar{\theta}\left|\psi_{0}\right\rangle=\left(1-\frac{[2]}{4} \theta \bar{\theta}\right)\left|\psi_{0}\right\rangle+\frac{\sqrt{[2]}}{2} \theta\left|\psi_{2}\right\rangle .
$$

Besides, using the dual operators $c$ and $\bar{c}$, one may consider the other set of the squeezing operators for para-Grassmannian pseudo Hermitian $q$-deformed systems as below

$$
\widetilde{S}(\theta)=\exp \left[\frac{1}{2}\left(\theta \bar{c}^{2}-\bar{\theta} c^{2}\right)\right],
$$

for three level system, the operators $c^{3}$ and $\bar{c}^{3}$ are zero, so the squeezing operator $\widetilde{S}(\theta)$ becomes

$$
\widetilde{S}(\theta)=I+\frac{1}{2}\left(\theta \bar{c}^{2}-\bar{\theta} c^{2}\right)-\frac{\theta \bar{\theta}}{8}\left(q^{2} \bar{c}^{2} c^{2}+c^{2} \bar{c}^{2}\right) .
$$

Hence, the squeezed state is given by

$$
|\tilde{\xi}\rangle=\widetilde{S}(\theta)\left|\phi_{0}\right\rangle .
$$


Using (4.1) we have

$$
|\tilde{\xi}\rangle=\left|\phi_{0}\right\rangle+\frac{\sqrt{[2]}}{2} \theta\left|\phi_{2}\right\rangle-\frac{[2]}{8} \theta \bar{\theta}\left|\phi_{0}\right\rangle=\left(1-\frac{[2]}{4} \theta \bar{\theta}\right)\left|\phi_{0}\right\rangle+\frac{\sqrt{[2]}}{2} \theta\left|\phi_{2}\right\rangle .
$$

It is notable that, the dual squeezing operator $|\tilde{\xi}\rangle$ can be obtained from the action of $\eta$ on $|\xi\rangle$. This means

$$
|\tilde{\xi}\rangle=\eta|\xi\rangle=\left(1-\frac{[2]}{4} \theta \bar{\theta}\right)\left|\phi_{0}\right\rangle+\frac{\sqrt{[2]}}{2} \theta\left|\phi_{2}\right\rangle .
$$

\subsection{Time evolution of squeezed states}

In analogy with coherent states, it would be worthy to study the time evolution of squeezed states. The time evolution of a system is governed by its Hamiltonian. Here, the time evolution of the three level squeezed states will be considered and it will be shown that the three level squeezed state remains squeezed state temporally. For a three level system we have obtained the squeezed state as

$$
|\xi\rangle=\left(1-\frac{[2]}{4} \theta \bar{\theta}\right)\left|\psi_{0}\right\rangle+\frac{\sqrt{[2]}}{2} \theta\left|\psi_{2}\right\rangle .
$$

Now, applying the time evolution operator on this state we have

$$
|\xi, t\rangle=e^{-i H t}|\xi\rangle=\left(1-\frac{[2]}{4} \theta \bar{\theta}\right) e^{-i E_{0} t}\left|\psi_{0}\right\rangle+\frac{\sqrt{[2]}}{2} \theta e^{-i E_{2} t}\left|\psi_{2}\right\rangle,
$$

or equivalently, it can be rewritten as

$$
|\xi, t\rangle=e^{-i E_{0} t} S(\theta(t))\left|\psi_{0}\right\rangle, \quad \text { where } \quad \theta(t)=e^{-i\left(E_{2}-E_{0}\right) t} \theta .
$$

Consequently, the time evolved state $|\xi, t\rangle$ is squeezed state. We note that, without loss of generality, one may take $E_{0}=0$, and write the time evolved squeezed state $|\xi, t\rangle$ as the action of the operator $S(\theta(t))$ on vacuum state $\left|\psi_{0}\right\rangle$. Similarly, for the dual space it can be deduced that, the time evolved squeezed state is

$$
|\tilde{\xi}\rangle=e^{-i H t}|\tilde{\xi}\rangle=e^{-i E_{0} t} \widetilde{S}(\theta)\left|\phi_{0}\right\rangle, \quad \text { where } \quad \theta(t)=e^{-i\left(E_{2}-E_{0}\right) t} \theta
$$

\subsection{Supersqueezed states}

Using the creation and annihilation operators for pseudo-Hermitian bosonic system we can define pseudo-Hermitian squeezing operator in analogy with the Hermitian squeezing operator. Hence, let us define the pseudo-bosonic squeezing operator as para-Grassmannian

$$
S(\eta)=\exp \left[\frac{1}{2}\left(\eta a^{\sharp}-\eta^{*} a^{2}\right)\right],
$$

where $\eta$ is a complex number, and $a, a^{\sharp}$ are bosonic pseudo-Hermitian operators. Hence the squeezed state for bosonic pseudo-Hermitian system becomes

$$
|\eta\rangle=S(\eta)\left|\psi_{0}\right\rangle=\exp \left[\frac{1}{2}\left(\eta a^{\sharp^{2}}-\eta^{*} a^{2}\right)\right]\left|\psi_{0}\right\rangle .
$$

In addition

$$
|\eta\rangle^{\prime}=S^{\prime}(\eta)\left|\phi_{0}\right\rangle=\exp \left[\frac{1}{2}\left(\eta a^{\dagger^{2}}-\eta^{*} \tilde{a}^{2}\right)\right]\left|\phi_{0}\right\rangle .
$$


Considering these states, and the squeezed states introduced for para-Grassmann variables one may study the structure of the supersymmetric squeezed states as the tensor product of the pseudo-bosonic and para-Grassmannian squeezed state

$$
|\eta, \theta\rangle=|\eta\rangle \otimes|\zeta\rangle=S(\eta, \zeta)\left|\psi_{0}\right\rangle \otimes\left|\psi_{0}\right\rangle
$$

where the supersqueezing operator is

$$
S(\eta, \theta)=S(\eta) \otimes S(\theta)=\exp \left[\frac{1}{2}\left(\eta a^{\sharp 2}-\eta^{*} a^{2}\right)\right] \otimes \exp \left[\frac{1}{2}\left(\theta \bar{b}^{2}-\bar{\theta} b^{2}\right)\right] .
$$

As mentioned for supercoherent states, there are some other possibilities in order to define supersqueezed states for the pseudo-Hermitian systems. Since, one may consider

$$
\left|\eta^{\prime}, \theta\right\rangle=|\eta\rangle^{\prime} \otimes|\zeta\rangle=S\left(\eta^{\prime}, \zeta\right)\left|\phi_{0}\right\rangle \otimes\left|\psi_{0}\right\rangle,
$$

where

$$
S\left(\eta^{\prime}, \theta\right)=S^{\prime}(\eta) \otimes S(\theta)=\exp \left[\frac{1}{2}\left(\eta a^{\dagger^{2}}-\eta^{*} \tilde{a}^{2}\right)\right] \otimes \exp \left[\frac{1}{2}\left(\theta \bar{b}^{2}-\bar{\theta} b^{2}\right)\right] .
$$

Also

$$
|\eta, \widetilde{\theta}\rangle=|\eta\rangle \otimes \widetilde{|\zeta\rangle}=S(\eta, \widetilde{\zeta})\left|\psi_{0}\right\rangle \otimes\left|\phi_{0}\right\rangle
$$

where

$$
S(\eta, \widetilde{\theta})=S(\eta) \otimes \widetilde{S(\theta)}=\exp \left[\frac{1}{2}\left(\eta a^{\sharp}-\eta^{*} a^{2}\right)\right] \otimes \exp \left[\frac{1}{2}\left(\theta \bar{c}^{2}-\bar{\theta} c^{2}\right)\right] .
$$

Finally, using the squeezed state for dual spaces the forthcoming super squeezed state can be obtained

$$
\left|\eta^{\prime}, \widetilde{\theta}\right\rangle=|\eta\rangle^{\prime} \otimes \widetilde{\zeta \zeta}=S\left(\eta^{\prime}, \widetilde{\zeta}\right)\left|\phi_{0}\right\rangle \otimes\left|\phi_{0}\right\rangle,
$$

here the squeezing operator is

$$
S\left(\eta^{\prime}, \widetilde{\theta}\right)=S^{\prime}(\eta) \otimes \widetilde{S(\theta)}=\exp \left[\frac{1}{2}\left(\eta a^{\dagger^{2}}-\eta^{*} \tilde{a}^{2}\right)\right] \otimes \exp \left[\frac{1}{2}\left(\theta \bar{c}^{2}-\bar{\theta} c^{2}\right)\right] .
$$

\section{Entanglement}

In this section, the entanglement of the pseudo-Hermitian coherent states is investigated. In fact, this is the generalization of $[10,11]$, to the states studied in this work. In those references, considering a proper linear combination of the tensor product of the multi-level coherent states as

$$
|\psi\rangle=\sum_{i_{1}, i_{2}, \ldots, i_{n}} f_{i_{1}, i_{2}, \ldots, i_{n}}\left|\theta_{i_{1}}\right\rangle\left|\theta_{i_{2}}\right\rangle \cdots\left|\theta_{i_{n}}\right\rangle
$$

and applying an appropriate weight function, it has been shown that, it is possible to get the right hand side of the following equation to be an entangled state, like Bell, cluster type, GHZ and $\mathbf{W}$ states, i.e.

$$
\int d \theta_{i_{1}} d \theta_{i_{2}} \cdots d \theta_{i_{n}} w\left(\theta_{i_{1}}, \ldots, \theta_{i_{n}}\right)|\psi\rangle=|\gamma\rangle,
$$

where $w\left(\theta_{i_{1}}, \ldots, \theta_{i_{n}}\right)$ is a proper weight function, and the state $|\gamma\rangle$, is the entangled state that we were going to obtain. The weight function is not unique and of course for a given state there may not be such a function at all. Pursuing this method, one can see that it is possible to construct entangled pseudo-Hermitian states. Since for the pseudo-Hermitian systems we deal with two sets of basis and consequently two set of coherent states, the possibility of superposing of the states is more than Hermitian coherent states. 


\section{$5.1 \quad$ Multi-qubit states}

Considering (3.1) and (3.2) for two level system $\left(q^{2}=-1\right)$ we get

$$
b \bar{b}+\bar{b} b=1 \quad \text { and } \quad c \bar{c}+\bar{c} c=1 .
$$

For a two level system para-Grassmann variables reduce to usual Grassman variables by the commutation relation

$$
\left\{\theta_{i}, \theta_{j}\right\}=0 \quad \Longrightarrow \quad \theta_{i}^{2}=0 .
$$

Thus, the $q$-deformed coherent states based on these operators become

$$
|\theta\rangle=\left|\psi_{0}\right\rangle-\theta\left|\psi_{1}\right\rangle, \quad \widetilde{|\theta\rangle}=\left|\phi_{0}\right\rangle-\theta\left|\phi_{1}\right\rangle
$$

Now using these states we can construct two level entangled states. For example, up to the normalization factors, the following pseudo-Hermitian version of the Bell states can be obtained. Since these states remind the well-known Bell states, one may call them pseudo-Hermitian Bell states (PBell states)

$$
\begin{aligned}
& \int d \theta|\mp \theta\rangle|-\theta\rangle=\left|\psi_{0}\right\rangle\left|\psi_{1}\right\rangle \pm\left|\psi_{1}\right\rangle\left|\psi_{0}\right\rangle, \\
& \int d \theta \widetilde{\mp \theta\rangle \mid \widetilde{-\theta}}=\left|\phi_{0}\right\rangle\left|\phi_{1}\right\rangle \pm\left|\phi_{1}\right\rangle\left|\phi_{0}\right\rangle, \\
& \left.\int d \theta|\mp \theta\rangle|\widetilde{-\theta\rangle}=| \psi_{0}\right\rangle\left|\phi_{1}\right\rangle \pm\left|\psi_{1}\right\rangle\left|\phi_{0}\right\rangle, \\
& \left.\int d \theta \widetilde{|\mp \theta\rangle \mid}-\theta\right\rangle=\left|\phi_{0}\right\rangle\left|\psi_{1}\right\rangle \pm\left|\phi_{1}\right\rangle\left|\psi_{0}\right\rangle .
\end{aligned}
$$

Also we have

$$
\begin{aligned}
& \int d \theta_{2} d \theta_{1}\left(1+\theta_{1} \theta_{2}\right)\left|\theta_{1}\right\rangle\left| \pm \theta_{2}\right\rangle=\left|\psi_{0}\right\rangle\left|\psi_{0}\right\rangle \pm\left|\psi_{1}\right\rangle\left|\psi_{1}\right\rangle, \\
& \left.\int d \theta_{2} d \theta_{1}\left(1+\theta_{1} \theta_{2}\right)\left|\widetilde{\left.\theta_{1}\right\rangle\left|\widetilde{\mp \theta_{2}}\right\rangle}=\right| \phi_{0}\right\rangle\left|\phi_{0}\right\rangle \pm\left|\phi_{1}\right\rangle\left|\phi_{1}\right\rangle, \\
& \int d \theta_{2} d \theta_{1}\left(1+\theta_{1} \theta_{2}\right)\left|\theta_{1}\right\rangle \widetilde{\left.\mp \theta_{2}\right\rangle}=\left|\psi_{0}\right\rangle\left|\phi_{0}\right\rangle \pm\left|\psi_{1}\right\rangle\left|\phi_{1}\right\rangle, \\
& \left.\int d \theta_{2} d \theta_{1}\left(1+\theta_{1} \theta_{2}\right)\left|\widetilde{\left.\theta_{1}\right\rangle}\right| \mp \theta_{2}\right\rangle=\left|\phi_{0}\right\rangle\left|\psi_{1}\right\rangle \pm\left|\phi_{1}\right\rangle\left|\psi_{0}\right\rangle
\end{aligned}
$$

It is notable that the normalization factor can be included in weight function. One gets the general form of the $\mathbf{W}$ state as follows

$$
\begin{aligned}
& \int d \theta\left(\frac{-1}{\sqrt{N}}\right) \underbrace{|\theta\rangle|\theta\rangle \cdots|\theta\rangle}_{n \text { times }}=\left|\mathbf{W}_{1}^{(n)}\right\rangle, \\
& \left|\mathbf{W}_{1}^{(n)}\right\rangle=\frac{1}{\sqrt{N}}\left(\left|\psi_{1} \psi_{0} \psi_{0} \cdots \psi_{0}\right\rangle+\left|\psi_{0} \psi_{1} \psi_{0} \cdots \psi_{0}\right\rangle+\cdots+\left|\psi_{0} \cdots \psi_{0} \psi_{0} \psi_{1}\right\rangle\right),
\end{aligned}
$$

here, $\frac{1}{\sqrt{N}}$ is the normalization factor of the state $\left|\psi_{1} \psi_{0} \psi_{0} \cdots \psi_{0}\right\rangle+\left|\psi_{0} \psi_{1} \psi_{0} \cdots \psi_{0}\right\rangle+\cdots+$ $\left|\psi_{0} \cdots \psi_{0} \psi_{0} \psi_{1}\right\rangle$. We can obtain the other states of this family like above. For instance,

$$
\int d \theta\left(\frac{-1}{\sqrt{N}}\right) \underbrace{\widetilde{|\theta\rangle}|\theta\rangle \cdots|\theta\rangle}_{n \text { times }}=\left|\mathbf{W}_{2}^{(n)}\right\rangle,
$$




$$
\left|\mathbf{W}_{2}^{(n)}\right\rangle=\frac{1}{\sqrt{N}}\left(\left|\phi_{1} \psi_{0} \psi_{0} \cdots \psi_{0}\right\rangle+\left|\phi_{0} \psi_{1} \psi_{0} \cdots \psi_{0}\right\rangle+\cdots+\left|\phi_{0} \cdots \psi_{0} \psi_{0} \psi_{1}\right\rangle\right)
$$

Likewise, we can obtain these states

$$
\begin{aligned}
\left|\mathbf{W}_{3}^{(n)}\right\rangle & =\frac{1}{\sqrt{N}}\left(\left|\phi_{1} \phi_{0} \psi_{0} \cdots \psi_{0}\right\rangle+\left|\phi_{0} \phi_{1} \psi_{0} \cdots \psi_{0}\right\rangle+\cdots+\left|\phi_{0} \cdots \psi_{0} \psi_{0} \psi_{1}\right\rangle\right), \\
\left|\mathbf{W}_{4}^{(n)}\right\rangle & =\frac{1}{\sqrt{N}}\left(\left|\phi_{1} \phi_{0} \phi_{0} \cdots \psi_{0}\right\rangle+\left|\phi_{0} \phi_{1} \phi_{0} \cdots \psi_{0}\right\rangle+\cdots+\left|\phi_{0} \cdots \psi_{0} \psi_{0} \psi_{1}\right\rangle\right), \\
\left|\mathbf{W}_{k}^{(n)}\right\rangle & =\frac{1}{\sqrt{N}}\left(\left|\phi_{1} \phi_{0} \phi_{0} \cdots \phi_{0}\right\rangle+\left|\phi_{0} \phi_{1} \phi_{0} \cdots \phi_{0}\right\rangle+\cdots+\left|\phi_{0} \cdots \phi_{0} \phi_{0} \phi_{1}\right\rangle\right)
\end{aligned}
$$

Likewise, we can construct the general forms of the $\mathbf{G H Z}$ state as follows

$$
\int d \theta_{1} d \theta_{2} \cdots d \theta_{n} w\left|\theta_{n}\right\rangle\left|\theta_{n-1}\right\rangle \cdots\left|\theta_{1}\right\rangle=\frac{1}{\sqrt{2}}\left(\left|\psi_{0} \psi_{0} \cdots \psi_{0}\right\rangle+\left|\psi_{1} \psi_{1} \cdots \psi_{1}\right\rangle\right)=\left|\mathbf{G H Z}_{1}^{(n)}\right\rangle,
$$

and

$$
\left.\int d \theta_{1} d \theta_{2} \cdots d \theta_{n} w\left|\widetilde{\left.\theta_{n}\right\rangle}\right| \theta_{n-1}\right\rangle \cdots\left|\theta_{1}\right\rangle=\frac{1}{\sqrt{2}}\left(\left|\phi_{0} \psi_{0} \cdots \psi_{0}\right\rangle+\left|\phi_{1} \psi_{1} \cdots \psi_{1}\right\rangle\right)=\left|\mathbf{G H Z}_{2}^{(n)}\right\rangle,
$$

and also

$$
\int d \theta_{1} d \theta_{2} \cdots d \theta_{n} w \widetilde{\left|\theta_{n}\right\rangle} \widetilde{\left.\theta_{n-1}\right\rangle} \cdots \widetilde{\left|\theta_{1}\right\rangle}=\frac{1}{\sqrt{2}}\left(\left|\phi_{0} \phi_{0} \cdots \phi_{0}\right\rangle+\left|\phi_{1} \phi_{1} \cdots \phi_{1}\right\rangle\right)=\left|\mathbf{G H Z}_{k}^{(n)}\right\rangle,
$$

where the weight function $w$ for the above states is

$$
w=\frac{1}{\sqrt{2}}\left((-1)^{\left[\frac{n}{2}\right]}+\theta_{n} \theta_{n-1} \cdots \theta_{1}\right) .
$$

It is notable that, when the pseudo-Hermitian system reduces to the hermitian one $(\eta=1)$, the pseudo-Hermitian entangled states reduces to the well-known maximally entangled states of the Hermitian systems, like maximally entangled Bell, W and GHZ. So fore instance the states $\left|\mathbf{W}_{i}^{(n)}\right\rangle$ reduce to just one maximally entangled state.

\subsection{Multi-qutrit states}

Using three level coherent states, it would be possible to construct entangled qutrit states. Since

for three level systems the operators $b^{3}$ and $\bar{b}^{3}$ vanish, the expansion of the equation (3.5) reduces to the following three level coherent state

$$
|\theta\rangle=\left|\psi_{0}\right\rangle+\frac{q^{2}}{\sqrt{[1] !}} \theta\left|\psi_{1}\right\rangle+\frac{\theta^{2}}{\sqrt{[2] !}}\left|\psi_{2}\right\rangle .
$$

Obviously, the para-Grassmann number $\theta$ is nilpotent, where $\theta^{3}=0$, and also $\left(q^{2}\right)^{3}=1$. Now this coherent state will be used to construct some entangled qutrit states. Here we introduce the construction of generalized Bell states for pseudo-Hermitian systems. The generalized Bell states for three level Hermitian systems are

$$
\begin{aligned}
& \left|\psi_{ \pm}\right\rangle=\frac{1}{\sqrt{3}}(|00\rangle \pm|11\rangle+|22\rangle), \\
& \left|\varphi_{ \pm}\right\rangle=\frac{1}{\sqrt{3}}(|02\rangle \pm|11\rangle+|20\rangle) .
\end{aligned}
$$


Here, we try to create the pseudo-Hermitian version of these states by using three level paraGrassmannian coherent states

$$
\begin{aligned}
& \int d \bar{\theta} d \theta w|\theta\rangle|\bar{\theta}\rangle=\left|\psi_{0}\right\rangle\left|\psi_{0}\right\rangle \pm\left|\psi_{1}\right\rangle\left|\psi_{1}\right\rangle+\left|\psi_{2}\right\rangle\left|\psi_{2}\right\rangle, \\
& \int d \bar{\theta} d \theta w|\widetilde{\theta}\rangle|\bar{\theta}\rangle=\left|\phi_{0}\right\rangle\left|\psi_{0}\right\rangle \pm\left|\phi_{1}\right\rangle\left|\psi_{1}\right\rangle+\left|\phi_{2}\right\rangle\left|\psi_{2}\right\rangle, \\
& \int d \bar{\theta} d \theta w|\theta\rangle|\widetilde{\bar{\theta}}\rangle=\left|\psi_{0}\right\rangle\left|\phi_{0}\right\rangle \pm\left|\phi_{1}\right\rangle\left|\psi_{1}\right\rangle+\left|\phi_{2}\right\rangle\left|\psi_{2}\right\rangle, \\
& \left.\int d \bar{\theta} d \theta w \widetilde{\theta}\right\rangle|\widetilde{\theta}\rangle=\left|\phi_{0}\right\rangle\left|\phi_{0}\right\rangle \pm\left|\phi_{1}\right\rangle\left|\phi_{1}\right\rangle+\left|\phi_{2}\right\rangle\left|\phi_{2}\right\rangle,
\end{aligned}
$$

where, for all above states

$$
w=\frac{\theta^{2} \bar{\theta}^{2}}{[2] !} \pm \frac{\theta \bar{\theta}}{[1] !}+q^{2}
$$

One may compare the above pseudo-Hermitian entangled states, with maximally entangled generalized Bell state (5.1). Note that in the case $\eta=1\left(\phi_{i}=\psi_{i}\right)$, all the states reduce to (5.1). Moreover, we have the following pseudo-Hermitian entangled states

$$
\begin{aligned}
& \int d \bar{\theta} d \theta w|\theta\rangle|\bar{\theta}\rangle=\left|\psi_{0}\right\rangle\left|\psi_{2}\right\rangle \pm\left|\psi_{1}\right\rangle\left|\psi_{1}\right\rangle+\left|\psi_{2}\right\rangle\left|\psi_{0}\right\rangle, \\
& \int d \bar{\theta} d \theta w|\widetilde{\theta}\rangle|\bar{\theta}\rangle=\left|\phi_{0}\right\rangle\left|\psi_{2}\right\rangle \pm\left|\phi_{1}\right\rangle\left|\psi_{1}\right\rangle+\left|\phi_{2}\right\rangle\left|\psi_{0}\right\rangle, \\
& \int d \bar{\theta} d \theta w|\theta\rangle|\widetilde{\bar{\theta}}\rangle=\left|\psi_{0}\right\rangle\left|\phi_{2}\right\rangle \pm\left|\phi_{1}\right\rangle\left|\psi_{1}\right\rangle+\left|\phi_{2}\right\rangle\left|\psi_{0}\right\rangle, \\
& \left.\int d \bar{\theta} d \theta w \widetilde{\theta}\right\rangle|\widetilde{\bar{\theta}}\rangle=\left|\phi_{0}\right\rangle\left|\phi_{2}\right\rangle \pm\left|\phi_{1}\right\rangle\left|\phi_{1}\right\rangle+\left|\phi_{2}\right\rangle\left|\phi_{0}\right\rangle,
\end{aligned}
$$

where

$$
w=\frac{\theta^{2}}{\sqrt{[2] !}} \pm \frac{\theta \bar{\theta}}{[1] !}+q^{2} \frac{\bar{\theta}^{2}}{\sqrt{[2] !}} .
$$

These states can be compared with maximally entangled generalized Bell state (5.2). Of course the weight function may be chosen in a way that we get entangled states in the subspaces. For instance,

$$
\begin{aligned}
& \int d \bar{\theta} d \theta\left(\frac{\theta^{2} \bar{\theta}^{2}}{[2] !}+q^{2}\right)|\theta\rangle|\bar{\theta}\rangle=\left|\psi_{0}\right\rangle\left|\psi_{0}\right\rangle+\left|\psi_{2}\right\rangle\left|\psi_{2}\right\rangle, \\
& \left.\int d \bar{\theta} d \theta\left(\frac{\theta^{2} \bar{\theta}^{2}}{[2] !}+q^{2}\right) \widetilde{\theta}\right\rangle|\widetilde{\bar{\theta}}\rangle=\left|\phi_{0}\right\rangle\left|\phi_{0}\right\rangle+\left|\phi_{2}\right\rangle\left|\phi_{2}\right\rangle .
\end{aligned}
$$

\subsection{Multi-qudit entangled states}

Now, using the coherent state obtained in (3.5), the entangled states for $n$ level pseudo-Hermitian systems will be considered. To this aim starting with the coherent state

$$
|\theta\rangle=\sum_{n=0}^{p} \frac{q^{n(n+1)}}{\sqrt{[n] !}} \theta^{n}\left|\psi_{n}\right\rangle,
$$

the product of two coherent states with para-Grassmann numbers $\theta$ and $\bar{\theta}$ reads

$$
|\theta\rangle|\bar{\theta}\rangle=\sum_{i, j=0}^{p} c_{i j} \theta^{i} \bar{\theta}^{j}\left|\psi_{i}\right\rangle\left|\psi_{j}\right\rangle,
$$


where

$$
c_{i j}=\frac{q^{(j-i)^{2}+(i+j)}}{\sqrt{[i] ![j] !}} .
$$

For instance, we may determine the weight function in a way that after integration the obtained state becomes

$$
\int d \bar{\theta} d \theta w|\theta\rangle|\bar{\theta}\rangle=\frac{1}{\sqrt{N}} \sum_{i=0}^{p}\left|\psi_{i}\right\rangle\left|\psi_{i}\right\rangle .
$$

Replacing the explicit form of the coherent states we get

$$
\int d \theta_{1} d \theta_{2} w \sum_{i, j=0}^{n-1} c_{i j} \theta_{1}^{i} \theta_{2}^{j}\left|\psi_{i}\right\rangle\left|\psi_{j}\right\rangle=\frac{1}{\sqrt{N}} \sum_{i=0}^{p}\left|\psi_{i}\right\rangle\left|\psi_{i}\right\rangle .
$$

Taking the general form of the weight function

$$
w=\sum_{k, l=0}^{p} w_{k, l} \bar{\theta}^{k} \theta^{l}
$$

and putting this weight in (5.4) and taking into account the quantization and the integration rules of para-Grassmannian variables we get

$$
\begin{aligned}
& \sum_{k, l=0}^{p} \sum_{i, j=0}^{p} c_{i j} w_{k, l} \int d \bar{\theta} d \theta \bar{\theta}^{k} \theta^{l+i} \bar{\theta}^{j}\left|\psi_{i}\right\rangle\left|\psi_{j}\right\rangle=\frac{1}{\sqrt{N}} \sum_{i=0}^{p}\left|\psi_{i}\right\rangle\left|\psi_{i}\right\rangle, \\
& \sum_{k, l=0}^{p} \sum_{i, j=0}^{p} c_{i j} w_{k, l} \bar{q}^{2 k(l+i)} \int d \bar{\theta} d \theta \theta^{l+i} \bar{\theta}^{k+j}\left|\psi_{i}\right\rangle\left|\psi_{j}\right\rangle=\frac{1}{\sqrt{N}} \sum_{i=0}^{p}\left|\psi_{i}\right\rangle\left|\psi_{i}\right\rangle, \\
& \sum_{k, l=0}^{p} \sum_{i, j=0}^{p} c_{i j} w_{k, l} \bar{q}^{2 k(l+i)} \delta_{p}^{l+i} \delta_{p}^{k+j}[p] !\left|\psi_{i}\right\rangle\left|\psi_{j}\right\rangle=\frac{1}{\sqrt{N}} \sum_{i=0}^{p}\left|\psi_{i}\right\rangle\left|\psi_{i}\right\rangle,
\end{aligned}
$$

where the symbol $\delta_{j}^{i}$ is the usual Kronecker delta. Note that

$$
\delta_{p}^{l+i} \delta_{p}^{k+j} \neq 0 \quad \Longrightarrow \quad l+i=p=k+j .
$$

From the right hand side if the the equation (5.5) it is clear that the terms with $i \neq j$ have to vanish in the left hand side of the equation, which due to (5.6) we get $w_{k, l}=0$ for $k \neq l$. With this description (5.5) gives

$$
\sum_{i=0}^{p} c_{i i} w_{p-i, p-i} \bar{q}^{2 p(p-i)}[p] !\left|\psi_{i}\right\rangle\left|\psi_{i}\right\rangle=\frac{1}{\sqrt{N}} \sum_{i=0}^{p}\left|\psi_{i}\right\rangle\left|\psi_{i}\right\rangle .
$$

Thus,

$$
w=\frac{1}{\sqrt{N}} \sum_{k=0}^{p} c_{(p-k),(p-k)}^{-1} \frac{q^{2 p k}}{[p] !} \bar{\theta}^{k} \theta^{k} .
$$

With this weight (5.3) holds. Using this weight function the below entangled states can be constructed

$$
\left.\int d \bar{\theta} d \theta w \widetilde{\theta \theta}\left|\widetilde{\bar{\theta}\rangle}=\frac{1}{\sqrt{N}} \sum_{i=0}^{p}\right| \phi_{i}\right\rangle\left|\phi_{i}\right\rangle .
$$

Of course the normalization factors $\left(\frac{1}{\sqrt{N}}\right)$ of the above states may not be the same. 


\section{Conclusion}

In conclusion, the $q$-deformed oscillator was generalized to pseudo-Hermitian systems and some of its important properties was studied. Introducing annihilation and creation operators for this system the new pseudo-Hermitian coherent and squeezed states were investigated. The overcompleteness property of the PGPHCSs examined. Also the stability of coherent and squeezed states were discussed. The pseudo-Hermitian supercoherent states as the product of a pseudoHermitian bosonic coherent state and a para-Grassmannian pseudo-Hermitian coherent state was introduced. This definition also was developed to define pseudo-Hermitian supersqueezed states. It was shown that, for $q$-oscillator algebra of $k+1$ degree of nilpotency based on the changed ladder operators, defined in here, we can obtain deformed $S U_{q^{2}}(2)$ and $S U_{q^{2 k}}(2)$ and also $S U_{q^{2 k}}(1,1)$. Finally, the entanglement of multi-level para-Grassmannian pseudo-Hermitian coherent state was considered. This was done by choosing an appropriate weight function, and integrating over tensor product of PGPHCSs. Thus, a relation between PGPHCSs and quantum entanglement was established. The entangled pseudo-Hermitian qubit states based on two level coherent states was given, and it was generalized for qutrit and qudit cases in general.

\section{References}

[1] Bender C.M., Boettcher S., Real spectra in non-Hermitian Hamiltonians having $\mathcal{P} \mathcal{T}$ symmetry, Phys. Rev. Lett. 80 (1998), 5243-5246, math-ph/9712001.

[2] Bender C.M., Boettcher S., Meisenger P.N., PT-symmetric quantum mechanics, J. Math. Phys. 40 (1999), 2201-2229, quant-ph/9809072.

[3] Bender C.M., Dunne G.V., Large-order perturbation theory for a non-Hermitian $\mathcal{P} \mathcal{T}$-symmetric Hamiltonian, J. Math. Phys. 40 (1999), 4616-4621, quant-ph/9812039.

[4] Mostafazadeh A., Pseudo-Hermiticity and generalized PT- and CPT-symmetries, J. Math. Phys. 44 (2003), 974-989, math-ph/0209018.

[5] Mostafazadeh A., Pseudo-Hermiticity versus PT symmetry: the necessary condition for the reality of the spectrum of a non-Hermitian Hamiltonian, J. Math. Phys. 43 (2002), 205-214, math-ph/0107001.

[6] Mostafazadeh A., Pseudo-Hermiticity versus PT-symmetry. II. A complete characterization of nonHermitian Hamiltonians with a real spectrum, J. Math. Phys. 43 (2002), 2814-2816, math-ph/0110016.

[7] Perlomov A., Generalized coherent states and their applications, Texts and Monographs in Physics, SpringerVerlag, Berlin, 1986.

[8] Fujii K., Introduction to coherent states and quantum information theory, quant-ph/0112090.

[9] Najarbashi G., Maleki Y., Maximal entanglement of two-qubit states constructed by linearly independent coherent states, Internat. J. Theoret. Phys. 50 (2011), 2601-2608, arXiv:1007.1387.

[10] Najarbashi G., Maleki Y., Entanglement of Grassmannian coherent states for multi-partite $n$-level systems, SIGMA 7 (2011), 011, 11 pages, arXiv:1008.4836.

[11] Najarbashi G., Maleki Y., Entanglement in multi-qubit pure fermionic coherent states, arXiv:1004.3703.

[12] Fu H., Wang X., Solomon A.I., Maximal entanglement of nonorthogonal states: classification, Phys. Lett. A 291 (2001), 73-76, quant-ph/0105099.

[13] Wang X., Sanders B.C., Multipartite entangled coherent states, Phys. Rev. A 65 (2001), 012303, 7 pages, quant-ph/0104011.

[14] Wang X., Bipartite entangled non-orthogonal states, J. Phys. A: Math. Gen. 35 (2002), 165-173, quant-ph/0102011.

[15] Wang X., Sanders B.C., Pan S.-H., Entangled coherent states for systems with $S U(2)$ and $S U(1,1)$ symmetries, J. Phys. A: Math. Gen. 33 (2000), 7451-7467, quant-ph/0001073.

[16] El Baz M., Hassouni Y., On the construction of generalized Grassmann representatives of state vectors, J. Phys. A: Math. Gen. 37 (2004), 4361-4368, math-ph/0409038.

[17] El Baz M., On the construction of generalized Grassmann coherent states, math-ph/0511028. 
[18] El Baz M., Hassouni Y., Madouri F., Z $Z_{3}$-graded Grassmann variables, parafermions and their coherent states, Phys. Lett. B 536 (2002), 321-326, math-ph/0206017.

[19] Cahill K.E., Glauber R.J., Density operators for fermions, Phys. Rev. A 59 (1999), 1538-1555, physics/9808029.

[20] Mansour T., Schork M., On linear differential equations with variable coefficients involving a paraGrassmann variable, J. Math. Phys. 51 (2010), 043512, 21 pages.

[21] Mansour T., Schork M., On linear differential equations involving a paragrassmann variable, SIGMA $\mathbf{5}$ (2009), 073, 26 pages, arXiv:0907.2584.

[22] Cabra D.C., Moreno E.F., Tanasa A., Para-Grassmann variables and coherent states, SIGMA 2 (2006), 087, 8 pages, hep-th/0609217.

[23] Trifonov D.A., Pseudo-boson coherent and Fock states, in Trends in Differential Geometry, Complex Analysis and Mathematical Physics, Editors K. Sekigawa et al., World Scientific, 2009, 241-250, arXiv:0902.3744.

[24] Cherbal O., Drir M., Maamache M., Trifonov D.A., Fermionic coherent states for pseudo-Hermitian two-level systems, J. Phys. A: Math. Theor. 40 (2007), 1835-1844, quant-ph/0608177.

[25] Najarbashi G., Fasihi M.A., Fakhri H., Generalized Grassmannian coherent states for pseudo-Hermitian n-level systems, J. Phys. A: Math. Theor. 43 (2010), 325301, 10 pages, arXiv:1007.1392.

[26] Daoud M., Hassouni Y., Kibler M., On generalized super-coherent states, Phys. Atomic Nuclei 61 (1998), 1821-1824, quant-ph/9804046.

[27] Daoud M., Kibler M., A fractional supersymmetric oscillator and its coherent states, math-ph/9912024.

[28] Nieto M.M., Coherent states and squeezed states, supercoherent states and supersqueezed states, in On Klauder's Path: a Field Trip, World Sci. Publ., River Edge, NJ, 1994, 147-155, hep-th/9212116.

[29] Nielsen M.A., Chuang I.L., Quantum computation and quantum information, Cambridge University Press, Cambridge, 2000.

[30] Petz D., Quantum information theory and quantum statistics, Springer-Verlag, Berlin, 2008.

[31] Majid S., Rodríguez-Plaza M.J., Random walk and the heat equation on superspace and anyspace, J. Math. Phys. 35 (1994), 3753-3760.

[32] Kerner R., $Z_{3}$-graded algebras and the cubic root of the supersymmetry translations, J. Math. Phys. 33 (1992), 403-411.

[33] Filippov A.T., Isaev A.P., Kurdikov A.B., Para-Grassmann differential calculus, Theoret. and Math. Phys. 94 (1993), 150-165, hep-th/9210075.

[34] Isaev A.P., Para-Grassmann integral, discrete systems and quantum groups, Internat. J. Modern Phys. A 12 (1997), 201-206, q-alg/9609030.

[35] Cugliandolo L.F., Lozano G.S., Moreno E.F., Schaposnik F.A., A note on Gaussian integrals over paraGrassmann variables, Internat. J. Modern Phys. A 19 (2004), 1705-1714, hep-th/0209172.

[36] Ilinski K.N., Kalinin G.V., Stepanenko A.S., $q$-functional Wick's theorems for particles with exotic statistics, J. Phys. A: Math. Gen. 30 (1997), 5299-5310, hep-th/9704181.

[37] El Baz M., Fresneda R., Gazeau J.P., Hassouni Y., Coherent state quantization of paragrassmann algebras, J. Phys. A: Math. Theor. 43 (2010), 385202, 15 pages, arXiv:1004.4706.

[38] Chaichian M., Demichev A.P., Polynomial algebras and higher spins, Phys. Lett. A 222 (1996), 14-20, hep-th/9602008.

[39] Daoud M., Kibler M., Fractional supersymmetric quantum mechanics as a set of replicas of ordinary supersymmetric quantum mechanics, Phys. Lett. A 321 (2004), 147-151, math-ph/0312019.

[40] Cherbal O., Drir M., Maamache M., Trifonov D.A., Supersymmetric extension of non-Hermitian su(2) Hamiltonian and supercoherent states, SIGMA 6 (2010), 096, 11 pages, arXiv:1009.5293. 